\title{
A Comparative Study of Flows Through Funnel-Shaped Bottlenecks Placed in the Middle and Corner
}

\author{
Hossein Tavana ${ }^{1} \cdot$ Kayvan Aghabayk $^{2} \cdot$ Karen Boyce $^{1}$ \\ ${ }^{1}$ Ulster University, FireSERT, Faculty of Computing, Engineering, and Built Environment, \\ Newtownabbey, UK \\ E-mail: tavanarezaei-h@ulster.ac.uk,ke.boyce@ulster.ac.uk \\ 2 University of Tehran, School of Civil Engineering, College of Engineering, University of \\ Tehran, Tehran, Iran \\ E-mail: Kayvan.aghabayk@ut.ac.ir
}

Received: 15 August 2021 / Last revision received: 21 October 2021 / Accepted: 2 November 2021

DOI: $10.17815 / C D .2021 .128$

\begin{abstract}
Upon exiting buildings, theatres, and stadiums, which house a great number of people, egress points can act as bottlenecks, resulting in crowded exits and decreased flows. Most studies investigating flow have been conducted in either narrow bottlenecks (doors) or funnel shape bottlenecks, with the latter investigating bottlenecks placed in the middle of the walkway. This study investigates, for the first time, crowd flow through funnel-shaped bottlenecks placed in the corner of the walkway and makes comparisons with similar bottlenecks of the same length, entrance and exit width placed in the middle of the walkway. The entry width and exit width of the bottlenecks were $3 \mathrm{~m}$ and $1 \mathrm{~m}$ respectively, with lengths varying from $1 \mathrm{~m}$ to $4 \mathrm{~m}$; they continued into a $10 \mathrm{~m}$ corridor. Ninety-four participants of various ages were observed moving through each of the configurations. The results indicated that using funnel-shaped bottlenecks in the middle of the walkway increased the flow rate significantly compared to the corner in bottlenecks with $2 \mathrm{~m}$ and $3 \mathrm{~m}$ lengths. This is contrary to what some other researchers have found for narrow bottlenecks placed in the middle and corner of a wall, although it is recognised that the configuration of funnel-shaped bottlenecks makes the comparison more complex and further work is required in this area. Notwithstanding these results are considered valuable for consideration when designing egress points and corridors in complex buildings such as metro and train stations.
\end{abstract}

Keywords Funnel shape bottleneck · exit position · exit configuration · crowd flow 


\section{Introduction}

The study of pedestrian movement and, in particular, an understanding of the role that bottlenecks play in emergency and non-emergency evacuation of a crowd is becoming more important as buildings and structures become more complex and house more and more people. Of particular importance in designing safe buildings, is an understanding of the impact of the location and dimensions of egress points on the characteristics of flow.

One of the most significant early studies on crowd movement was conducted by Togawa [1], who identified and presented relationships between speed and density and equations to predict evacuation time. In a similar study, Fruin [2] studied pedestrian movement in everyday circulation space, developing the concept of Levels of Service to characterise the movement in different densities and associated speeds and flows. Since then, many researchers have explored movement via controlled experiments [3-5], evacuation drills $[6,7]$, by analysing actual footage of everyday pedestrian circulation $[8,9]$, computer simulation and modelling $[10,11]$, in virtual reality $[12,13]$, and also by studying non-human organisms [14-16]. Of these methods, experimental investigation of crowd movement has proven to be the most popular, since it enables the conditions and geometries of the building components to be controlled [17].

One of the first studies that investigated exit flow in high density through bottlenecks of different widths was conducted by Muir et al. [18]. In this study, a series of controlled experiments were undertaken to understand the impact of different exit widths (between $0.6 \mathrm{~m}$ to $1.8 \mathrm{~m}$ ) and different numbers of participants on evacuation from an aircraft. In another experiment, Kretz et al. [19] explored the relationship between flow rate and the width of the egress point with 80-100 young participants moving through widths which varied from $0.4 \mathrm{~m}$ to $1.6 \mathrm{~m}$. It was concluded that, by increasing the width from 0.8 $\mathrm{m}$ to $1.2 \mathrm{~m}$, the flow rate increased from 1.43 to $2.15 \mathrm{p} / \mathrm{s}$ [19]. Seyfried et al. [3] also undertook controlled experiments with 20, 40, and 60 young and healthy participants moving through exit points of varying widths from $0.8 \mathrm{~m}$ to $1.2 \mathrm{~m}$ and drew the same conclusion, i.e. that the flow rate increased with increasing width. It is important to note that in each of these studies, the participants were young individuals, and most were students.

More recently, Daamen and Hoogendoorn [20] attempted to understand the effect of width on exit flow during emergencies by producing different whoop signals to simulate different levels of emergency. The examined widths were $0.5,0.85,1,1.1,1.65,2.2,2.75$ $\mathrm{m}$, and the results indicated that the flow rate was higher than $2.25 \mathrm{p} / \mathrm{m} / \mathrm{s}$ (the expected value of flow in the Dutch building code) in 13 out of the 16 experiments [20].

The aforementioned studies focussed on the dimensions of the egress points and, specifically, the relationship between exit width and flow. Other studies, however, have investigated the impact of the location of exit points on the flow, i.e. directly comparing the flows for corner exit points and centrally located exit points [16,21]. For example, Shiwakoti and Sarvi [22] investigated the movement of ants from an enclosed area via a corner and centrally located exit points and then scaled and simulated the evacuation of humans from a room with the exit points similarly located. They observed that, in both experiment and simulation, changing the location of the egress point from the middle to the corner of the 
walkway resulted in the evacuation time decreasing to less than half; the results of the simulation showed that evacuation times for the first 50 pedestrians from the middle and corner exits were 30.5 and $13.8 \mathrm{~s}$, respectively [22]. In another study, Lin et al. [23] explored the effect of different levels of stimuli on the evacuation of mice through centrally located bottlenecks. However, Chen et al. [21] reproduced the experiments conducted by Lin et al. [23] with the same breed of mice, and exit width but, additionally, changed the position of the egress point from the centre to the corner of the wall. Chen et al. [21] observed that the evacuation from corner exits was not affected significantly by the level of stimulus (mean evacuation time for each mouse was approximately $3.5 \mathrm{~s}$ in all conditions [21]) but in the case of the centrally located exit, the evacuation time varied from $2.5 \mathrm{~s}$ to $5.1 \mathrm{~s}$ as the stimulus was increased from low level to high level [21]. Both these studies suggested that the location of the bottleneck may impact evacuation time. However, although these studies were conducted on organisms with behaviour that is considered to be similar to humans, the results may not necessarily be generalisable to the evacuation of humans [24].

More recently, Jianyu et al. [25] conducted a series of experiments with 131 participants (college students, mean age 18.9 years) to investigate how the width and the location of exit points impacted flow. The results indicated that the flow rate through the centrally located exit point was higher than that through the corner exit point, but that the difference in flow rates decreased as the width of the exit points increased (from $0.6 \mathrm{~m}$ to $0.8 \mathrm{~m}$ to $1.0 \mathrm{~m}$ ). In another recent study, Shi et al. [24] investigated the effect of the location of the egress point and a nearby column on crowd flow. The subjects ( 26 healthy male and 24 healthy female students) participated in two scenarios in which they were instructed to move at 'normal walking speed' and 'slow running speed'. The results indicated that the corner egress points were more efficient i.e. had higher flow capacity than the centrally located egress points. For example, the flow rates for the middle and corner egress points without any obstacles at 'normal walking speed' were 2.67 and $2.81 \mathrm{p} / \mathrm{m} / \mathrm{s}$, respectively; the flow rates for the 'slow run' were 3.37 and $4.18 \mathrm{p} / \mathrm{m} / \mathrm{s}$, respectively.

It should be noted that all the aforementioned studies considered simple openings as egress points i.e. doors; however, simple changes in the architecture of the egress point may also increase its capacity [26,27]. In this regard, a number of studies have investigated the flow through other architectural adjustments of egress points, in particular funnel-shaped bottlenecks. For example, Oh and Park [28] conducted experiments with mice to investigate the impact of the configuration of the funnel-shaped bottleneck placed in the middle of the walkway on flow dynamics. They found that the evacuation time decreased by increasing the angle of the funnel from 0 to $75^{\circ}$ whilst the speed decreased. In this study, the most efficient angle of the funnel was $45^{\circ}$.

In another study, Sun et al. [26] conducted a series of experiments involving 50 healthy students (27 males and 23 females, mean age of 22 years) to understand and compare flows through a narrow bottleneck with $1 \mathrm{~m}$ width and funnel-shaped bottlenecks of $1 \mathrm{~m}$ narrow width where the width of entry was $5 \mathrm{~m}$ and the angle (between the entrance and wall extension) varied $\left(30^{\circ}, 45^{\circ}, 60^{\circ}\right.$, and $\left.90^{\circ}\right)$. They concluded that the most efficient bottleneck was the funnel-shaped bottleneck with an angle between $46^{\circ}$ and $65^{\circ}$. Another recent study by Pan et al. [29] investigated the impact of wheelchair users and 4 different 
angles of funnel-shaped bottleneck on evacuation flow. The narrow point of the bottleneck in each case was $1.2 \mathrm{~m}$ and the angles considered were $0^{\circ}$ (i.e. no funnel), $15^{\circ}, 30^{\circ}$, and $45^{\circ}$. Eighty-five healthy individuals (40 females and 45 males, mean age: 22 years) including $0,1,2,3$ wheelchair users were present in different runs. The most efficient angle in this study both without, and with just one, wheelchair user was $45^{\circ}$. They established, however, by increasing the number of wheelchair users, the angle was deemed to lose its benefits.

In a more extensive study on crowd flow through funnel-shaped bottlenecks, Tavana and Aghabayk [27] conducted a series of experiments with a wide age range of participants (10 to 70 years old). They compared the flow through a narrow bottleneck of 1 $\mathrm{m}$ width and funnel-shaped bottlenecks of different lengths (1-4 m) and widths (2-4 m), and hence different angles, and concluded that the funnel-shaped bottleneck with a $26.6^{\circ}$ angle was the most efficient.

To summarise, the aforementioned studies have demonstrated that funnel-shaped bottlenecks could indeed improve the crowd flow characteristics in an evacuation compared to simple openings. The potential benefits of funnel shape bottlenecks have also been recognised by Helbing et al. [4]. However, until now, all studies on funnel-shaped bottlenecks have considered the egress point to be centrally located. Furthermore, age is an important factor in crowd evacuation and may affect the flow rate [30], and it is important to note that most studies to date have been conducted by recruiting young participants (mostly students).

It is important, therefore, to further investigate how the architecture of egress points impacts the capacity of exit. This study will, therefore, investigate the flow through bottlenecks placed in the corner and compare it with those ones placed in the middle of the walkway for a more diverse sample of participants.

\section{Methodology}

\subsection{Experimental Design}

The main aim of this study was to open a new area of research on funnel-shaped bottlenecks and investigate the flow rates through the mentioned bottlenecks placed in the middle and corner of a walkway for a mixed age group.

The experiments were held on Thursday $26^{\text {th }}$ October 2017 at 10 am in the School of Civil Engineering, at the central campus of the University of Tehran. The experiment day was sunny, and the temperature was $24^{\circ} \mathrm{C}$.

The test area was designed to be $6 \mathrm{~m}$ wide and was bordered with tables and chairs. The bottlenecks were adjusted with stands (with $1.6 \mathrm{~m}$ height) which were taped in 0.6 $\mathrm{m}, 1.1 \mathrm{~m}$, and $1.6 \mathrm{~m}$ height to prevent pedestrians from going outside the path.

In total, 8 different funnel-shaped bottlenecks were investigated, four of which were centrally located in the $6 \mathrm{~m}$ wide walkway, Fig. 1(a), with the other four placed in the corner, Fig. 1(b). The large width and the small width of all bottlenecks were $3 \mathrm{~m}$ and 1 $\mathrm{m}$, respectively, and the length varied from $1 \mathrm{~m}$ to $4 \mathrm{~m}$ in $1 \mathrm{~m}$ intervals (which changed 


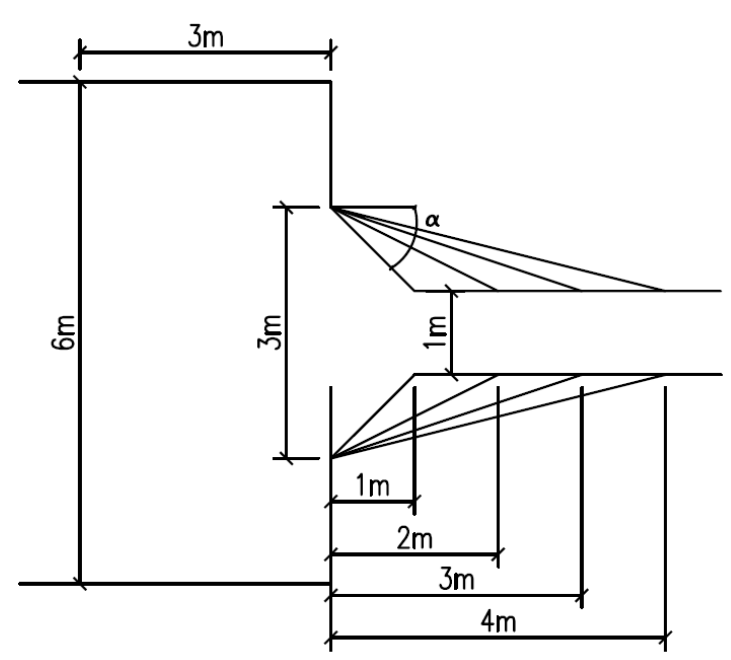

(a)

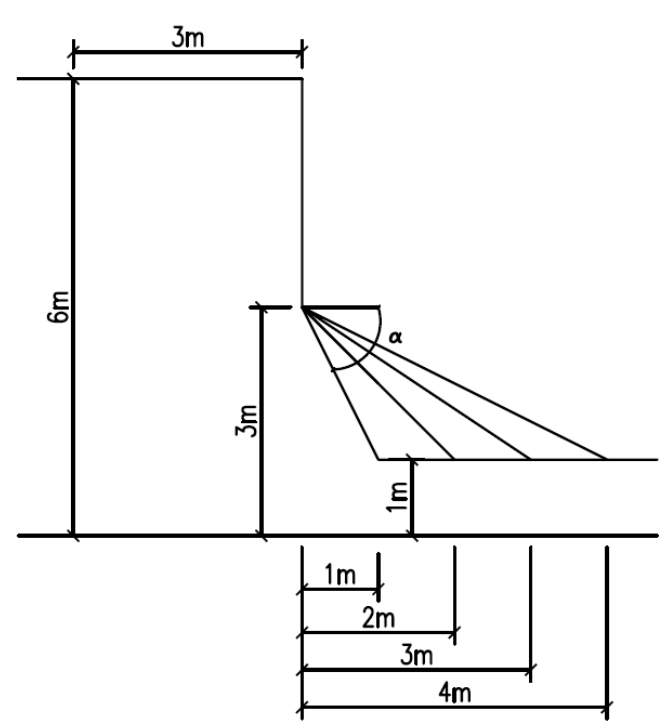

(b)

Figure 1 a) Funnel-shaped bottlenecks placed in the middle b) Funnel-shaped bottlenecks placed in the corner

the angle of the approach). For the centrally located bottlenecks, the angles were 45, 26.6, 18.4, and 14 degrees for 1, 2, 3, and 4 m bottleneck widths respectively, and for the corner bottlenecks, the angles were 63.4, 45, 33.7, 26.6 degrees, for the 1, 2, 3, and $4 \mathrm{~m}$ lengths of the bottlenecks, respectively. All bottlenecks were connected to a 1-metre wide corridor which continued for $10 \mathrm{~m}$.

The experiment was performed in two parts. The first set of trials involved evacuation via the centrally located bottlenecks. The participants were then given a break for a rest to prevent tiredness, after which time evacuation through the bottlenecks placed in the corner was investigated. Three runs were performed for each configuration i.e. in total 24 trials were recorded.

The experiment was recorded using a camera with HD quality in 60 frames/ second. The camera was mounted on an $8 \mathrm{~m}$ high crane. Specific points on the ground with known locations were used for calibrating and adjusting the location of the camera. After recording, the movies were converted to 30 frames/ second. Fig. 2 shows a screenshot of the experiment.

\subsection{Participants and Procedures}

The participants were healthy individuals who were recruited from family and friends of the experiment's organisers via an advertisement that explained the procedure of the experiment. During the recruitment process, a deliberate attempt was made to involve persons across a wide age range as might be expected in many typical evacuation contexts. Ninety-four individuals, i.e. 43 (45.7\%) males and 51 (54.3\%) females, aged 10-70 years 


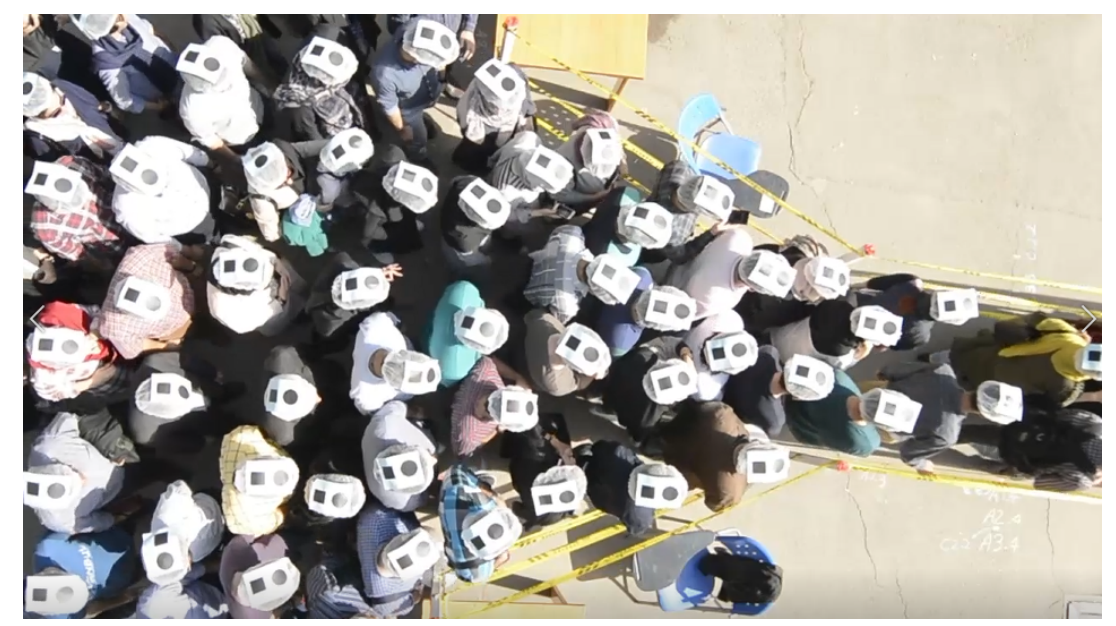

Figure 2 Screenshot of the Experiment

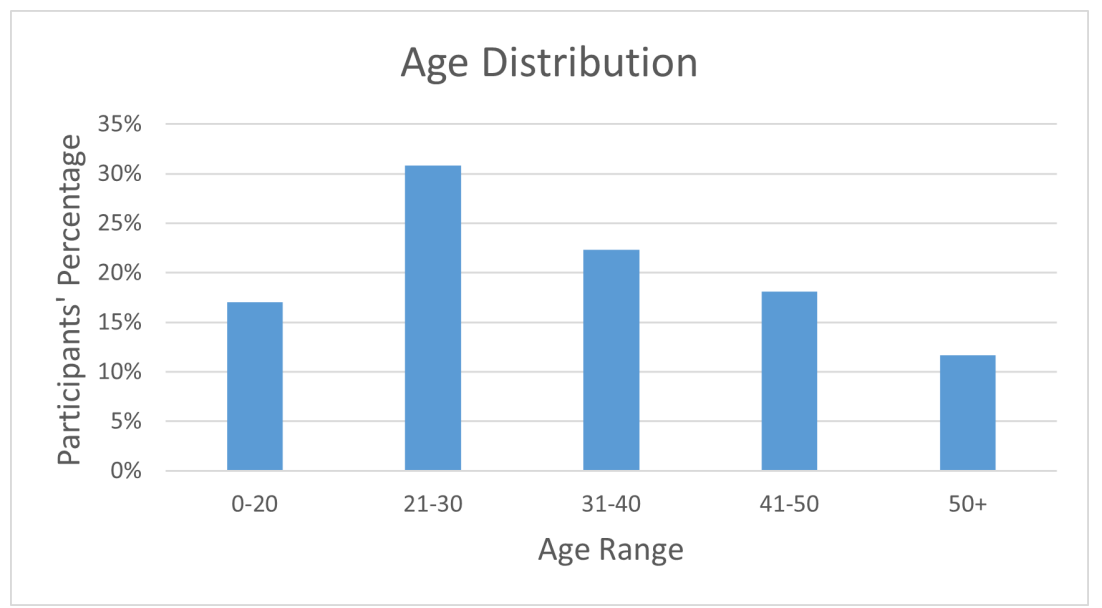

Figure 3 Age Distribution of Participants

volunteered to participate in this experiment. The age distribution of the participants is shown in Fig. 3. A professor, a graduate student, and three technical staff were present to manage the experiment.

In order to facilitate tracking, each individual was given a hat with indicators before the experiment, and participants were asked to walk at their normal speed i.e., to suppose that they were going to work, school, etc., on a typical day. Prior to the experiment, participants were asked to evacuate via a $1 \mathrm{~m}$ wide bottleneck to become familiar with the experimental setup and procedures. At the beginning of each trial, individuals were located approximately $3 \mathrm{~m}$ distance from the egress point with a density of 3.1 persons/ $\mathrm{m}^{2}$. This density was chosen to simulate a high-density evacuation as suggested in a study by Seyfried et al. [3]. 


\section{Results}

The aim of this study was to investigate the flow rate through the bottlenecks with different configurations and placed in different locations (corner and middle) of the walkway. This parameter can easily determine the efficiency of an egress point by showing the number of people who are able to pass the egress point during a time interval. To calculate the flow rate, a hypothetical line was considered at the end of the bottlenecks, i.e. just before the participants exited the bottleneck and entered the corridor, where the width was $1 \mathrm{~m}$. The flow rate in bottlenecks placed in the middle and the corner was calculated thus Eq. 1:

$$
J=N / \Delta t=\frac{N}{t_{n}-t_{1}}
$$

Where:

$\mathrm{N}$ is the number of individuals passing the line,

$\mathrm{t}_{1}$ is the time that the first pedestrian passed the line,

$t_{n}$ is the time that the last pedestrian passed the line.

The results for the bottlenecks placed in the middle are shown in Tab. 1. It can be seen that increasing the bottleneck length from $1 \mathrm{~m}$ to $2 \mathrm{~m}$ (with the associated decrease in angle from $45^{\circ}$ to $26.6^{\circ}$ ) resulted in a slight increase in flow from $2.13 \mathrm{p} / \mathrm{m} / \mathrm{s}$ to $2.28 \mathrm{p} / \mathrm{m} / \mathrm{s}$ (approximately 7\%). Subsequent increases in length (and associated reductions in angle) resulted in reductions in the flow (to $2.02 \mathrm{p} / \mathrm{m} / \mathrm{s}$ at length $4 \mathrm{~m}\left(14^{\circ}\right.$ angle)). However, the flow rate in the bottleneck with $3 \mathrm{~m}$ length $\left(18.4^{\circ}\right.$ angle) was greater than the bottleneck with $1 \mathrm{~m}$ length $\left(45^{\circ}\right.$ angle).

\begin{tabular}{lllll}
\hline Length $(\mathrm{m})$ & 1 & 2 & 3 & 4 \\
\hline Angle (Degree) & 45 & 26.6 & 18.4 & 14 \\
Flow (p/m/s) & 2.13 & 2.28 & 2.21 & 2.02 \\
\hline
\end{tabular}

Table 1 Average flow for bottlenecks placed in the middle

The results for the bottlenecks located in the corner are presented in Tab. 2.

\begin{tabular}{lllll}
\hline Length $(\mathrm{m})$ & 1 & 2 & 3 & 4 \\
\hline Angle (Degree) & 63.4 & 45 & 33.7 & 26.6 \\
Flow (p/m/s) & 1.99 & 1.97 & 2.03 & 2.08 \\
\hline
\end{tabular}

Table 2 Average flow for bottlenecks placed in the corner

Here we find a different trend, i.e. for bottlenecks placed in the corner, there was a slight decrease in flow (from $1.99 \mathrm{p} / \mathrm{m} / \mathrm{s}$ to $1.97 \mathrm{p} / \mathrm{m} / \mathrm{s}$ ) with an increase in length from $1 \mathrm{~m}$ to $2 \mathrm{~m}$ (reduction in angle from $63.4^{\circ}$ to $45^{\circ}$ ), followed by increases in flow with further increases in length to 3 and $4 \mathrm{~m}$ (angles of $33.7^{\circ}$ and $26.6^{\circ}$ respectively). 


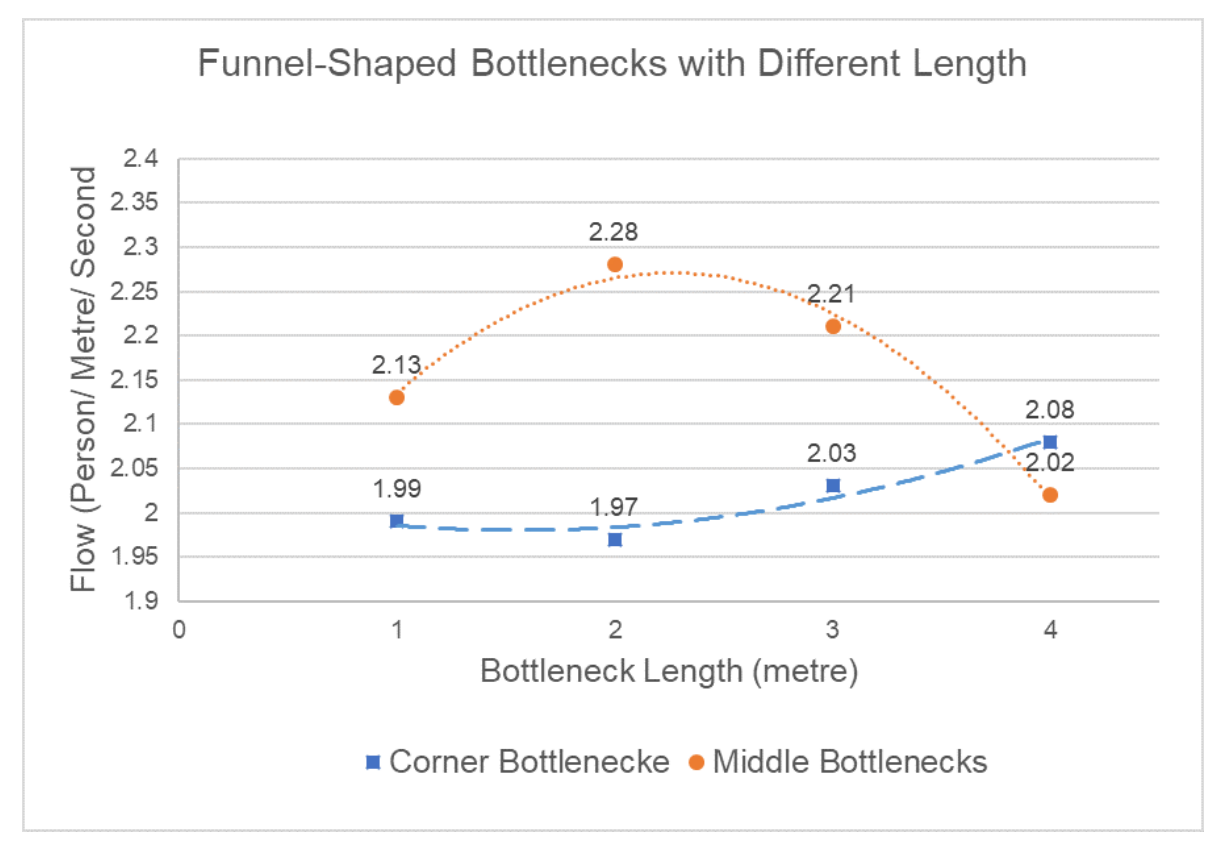

Figure 4 Diagram of flow vs. length of bottleneck for bottlenecks with different lengths

Fig. 4 compares the flow rate through bottlenecks in the middle and corner (orange circle represents middle bottlenecks and corner bottlenecks shown with blue square) for different lengths.

It is clear that in all configurations, except for the bottleneck of $4 \mathrm{~m}$ length, the flow through the centrally located bottlenecks was more efficient than through the respective corner bottlenecks. The change in flow between the middle and corner bottlenecks were $-6.6 \%,-13.5 \%,-8.1 \%$ and $+3 \%$ for lengths of $1,2,3$ and $4 \mathrm{~m}$ respectively. The greatest difference between the centrally placed and corner bottlenecks occurs in the condition of highest flow and lowest flow for the middle and corner bottlenecks respectively, i.e. at bottlenecks which are $2 \mathrm{~m}$ in length.

To investigate the significance of the differences in pedestrian flow for different dimensions within each configuration of the bottlenecks, i.e. corner and middle, the ANOVA test was used. The analyses were conducted in IBM SPSS Statistics (V 26) with a 95\% level of confidence. For the centrally located bottlenecks, there were significant differences between the bottlenecks of different lengths $(\mathrm{P}$-value $=0.018<0.05)$. The Bonferroni post hoc tests revealed that the flow rate in the centrally located bottleneck with $2 \mathrm{~m}$ length was significantly greater than the flow rate in the bottleneck with $4 \mathrm{~m}$ length $(\mathrm{P}$-value $=0.022$ $<0.05)$. Therefore, it can be concluded that by adjusting the angle, a higher flow rate can be achieved. On the other hand, for the corner bottlenecks, although the flow rate slightly increased with increases in length beyond $2 \mathrm{~m}$, these differences were not significant $(\mathrm{P}=$ 0.186).

To check the significance of the differences between corner and middle configurations of the bottleneck, T-test was used on each pair of bottlenecks of the same length, entry and exit width. The results of the test show that for $2 \mathrm{~m}$ and $3 \mathrm{~m}$ length, the flow rate in the 
middle bottlenecks was significantly greater than that in the corner bottlenecks ( $\mathrm{P}$-value $=$ 0.011 and 0.046 , respectively). On the other hand, no statistically significant differences were observed between the middle and corner bottlenecks for $1 \mathrm{~m}$ and $4 \mathrm{~m}$ lengths (P-value $=0.063$ and 0.179 , respectively).

\section{Discussion}

As mentioned previously, a number of experimental studies, involving both non-human organisms and human participants, and computer simulations have been conducted to explore the flow characteristics through bottlenecks of different configurations and dimensions. Experimental studies, involving mice or ants, usually found that the relocation of the bottlenecks from the middle of a wall to the corner increased the efficiency of the bottleneck [22,31,32]. The study by Shi et al. [24], which involved human participants evacuating at both 'normal' walking speed and 'slow running' speed, also established that the flow rate through the corner bottlenecks was higher than the equivalent bottleneck placed in the middle. However, some modelling studies have found different and, in some cases, opposite results of the influence of the location of egress points on evacuation parameters $[33,34]$.

In a similar story to investigate the effect of changing the narrow bottlenecks to funnelshaped bottlenecks on crowd evacuation, Oh and Park [28] explored the evacuation of mice and then simulated that to extract crowd evacuation; they found that by changing the configuration from narrow bottlenecks to funnel-shaped bottlenecks, the efficiency of the bottleneck increased. These results have been confirmed in experimental studies with human participants conducted by different researchers [26, 27, 29]. It should be mentioned, however, that in all these studies, the funnel-shaped bottlenecks with human participants were located in the middle of the walkway.

This study is the first study, therefore, to measure the differences between the crowd flow through funnel-shaped bottlenecks placed in the middle and corner of a walkway. By comparing the results of flow rates in the corner and middle funnel-shaped bottlenecks, it can be concluded that in three of the four adjustments the middle bottlenecks had a higher flow rate than those of equivalent length placed in the centre. In just one adjustment (the bottlenecks of $4 \mathrm{~m}$ in length), the corner bottleneck performed better. A T-test indicated that for the $2 \mathrm{~m}$ and $3 \mathrm{~m}$ lengths, the flow rate through the bottlenecks placed in the middle was significantly greater than the flow through the respective bottlenecks placed in the corner $(\mathrm{P}=0.011$ and $\mathrm{P}=0.046$ respectively). On the other hand, the observed differences between the flow rates for the middle and corner bottlenecks for $1 \mathrm{~m}$ and $4 \mathrm{~m}$ lengths were not significant $(\mathrm{P}=0.063$ and 0.179 , respectively).

It is not possible to fully explain the reasons for these differences. It is important, however, to recognise that, although the entry width and exit width of the respective bottlenecks in the middle and corner were equal, and the area within the funnel was also equal for a given length, the adjustments were different, i.e. the bottlenecks placed in the middle had a sloping entry to the narrow part of the bottleneck on both sides, whilst the bottlenecks in the corner had a sloping entry to the narrow part of the bottleneck on 
just one side and a direct entry alongside the wall on the other side. The more efficient flow in the centrally located funnel-shaped bottlenecks may simply be because there was more opportunity and space for the crowd to be more organised to enter the funnel from both sides compared to the respective cases in which the funnel was located in the corner. In comparison, a jam was observed to occur on one side of the funnel-shaped bottleneck placed in the corner and the efficiency of the egress point decreased. By increasing the length of the entrance for corner bottlenecks, more people were able to enter the bottleneck at a given time and indeed, the jam occurred inside the bottleneck whilst the borders had an influence on organising evacuees to exit. Therefore, rather than struggling to enter the bottleneck and overflowing the jam before the entrance, participants could organise their position in the bottleneck and overall, the crowd was organised more efficiently. As a result, the efficiency of the corner bottlenecks increased slightly with increased funnel length, although these differences were not found to be statistically significant.

\section{Conclusion}

In public places and structures with users both in daily commute and emergencies the egress points can act as bottlenecks and may cause congestion. It is, therefore, important to explore ways in which the flow and, consequently, the efficiency of bottlenecks can be increased.

This study, conducted at the University of Tehran, is the first study to directly compare the efficiency of funnel-shaped bottlenecks placed in the middle and corner of the walkway. It compared the flow of 94 volunteers (aged 11-70) through 4 funnel-shaped bottlenecks which varied in length from 1 to $4 \mathrm{~m}$; the entrance and exit widths were $3 \mathrm{~m}$ and $1 \mathrm{~m}$ respectively.

The results show that the flow through the centrally located funnel-shaped bottlenecks, for all the funnels apart from $4 \mathrm{~m}$ length, was higher than the flows through the respective bottlenecks placed in the corner. For bottlenecks of 2 and $3 \mathrm{~m}$ lengths, these differences were significant. Besides, increasing the length of the bottleneck located in the middle and corner of the walkway showed different trends.

This research investigated the flow rates through funnel-shaped bottlenecks with the same entry, exit width and length that were placed in the middle and corner of the walkway. Although the area within the funnels placed in the middle and the corner was the same, the configurations of the bottlenecks were different, and this may have contributed somewhat to the efficiency of the bottlenecks. It is suggested that further research is needed to fully appreciate how the location and configuration of the funnel-shaped bottleneck impacts flow capacity, i.e. further research should explore funnel-shaped bottlenecks with two sloped sides and how their capacity is impacted by their location on the walkway. Furthermore, the efficiency of funnel-shaped bottlenecks with a wider range of angles should be explored.

This study provides insights into the crowd flow and suggests further research on funnel-shaped bottlenecks placed in different locations. The results should be of interest and valuable for architectures, urban designers, and fire safety engineers to design corridors and egress points in complex structures e.g. metro stations. 


\section{References}

[1] Togawa K: Study on fire escapes basing on the observation of multitude currents: Building Research Institute. Ministry of Construction, Report No. 14 (1955).

[2] Fruin JJ: Pedestrian planning and design. No. 206 pp. (1971).

[3] Seyfried A, Passon O, Steffen B, Boltes M, Rupprecht T, Klingsch W: New insights into pedestrian flow through bottlenecks. Transportation Science vol. 43 (3), pp. 395-406 (2009), doi:10.1287/trsc.1090.0263

[4] Helbing D, Buzna L, Johansson A, Werner T: Self-organized pedestrian crowd dynamics: Experiments, simulations, and design solutions. Transportation Science vol. 39 (1), pp. 1-24 (2005), doi:10.1287/trsc.1040.0108

[5] Hoogendoorn SP, Daamen W: Pedestrian behavior at bottlenecks. Transportation Science vol. 39 (2), pp. 147-159 (2005), doi:10.1287/trsc. 1040.0102

[6] Peacock RD, Averill JD, Kuligowski ED: Stairwell evacuation from buildings: what we know we don't know. In: Pedestrian and evacuation dynamics 2008: Springer, pp. 55-66 (2010), doi:10.1007/978-3-642-04504-2_4

[7] Kuligowski E, Peacock R, Wiess E, Hoskins B: Stair evacuation of older adults and people with mobility impairments. Fire Safety Journal vol. 62, pp. 230-237 (2013), doi:10.1016/j.firesaf.2013.09.027

[8] Zhang X, Weng W, Yuan H, Chen J: Empirical study of a unidirectional dense crowd during a real mass event. Physica A: Statistical Mechanics and its Applications vol. 392(12), pp. 2781-2791 (2013), doi:10.1016/j.physa.2013.02.019

[9] Shiwakoti N: Understanding differences in emergency escape and experimental pedestrian crowd egress through quantitative comparison. International Journal of Disaster Risk Reduction vol. 20, pp. 129-137 (2016), doi:10.1016/j.ijdrr.2016.11.002

[10] Kirchner A, Klüpfel H, Nishinari K, Schadschneider A, Schreckenberg M: Simulation of competitive egress behavior: comparison with aircraft evacuation data. Physica A: Statistical Mechanics and its Applications vol. 324(3-4), pp. 689-697 (2003), doi:10.1016/S0378-4371(03)00076-1

[11] Blue VJ, Adler JL: Cellular automata microsimulation for modeling bi-directional pedestrian walkways. Transportation Research Part B: Methodological vol. 35(3), pp. 293-312 (2001), doi:10.1016/s0191-2615 (99) 00052-1 
[12] Moussaïd M, Kapadia M, Thrash T, Sumner RW, Gross M, Helbing D, et al.: Crowd behaviour during high-stress evacuations in an immersive virtual environment. Journal of The Royal Society Interfacel vol. 13(122), pp. 2016041420160414 (2016), doi:10.1098/rsif.2016.0414

[13] Ronchi E, Nilsson D, Kojić S, Eriksson J, Lovreglio R, Modig H, et al.: A virtual reality experiment on flashing lights at emergency exit portals for road tunnel evacuation. Fire technology vol. 52(3), pp. 623-647 (2016), doi:10.1007/s10694-015-0462-5

[14] Dias C, Sarvi M, Shiwakoti N, Ejtemai O, Burd M: Investigating collective escape behaviours in complex situations. Safety science vol. 60, pp. 87-94 (2013), doi:10.1016/j.ssci.2013.07.005

[15] Garcimartín A, Pastor J, Ferrer L, Ramos J, Martín-Gómez C, Zuriguel I: Flow and clogging of a sheep herd passing through a bottleneck. Physical Review E vol. 91(2), pp. 022808-022808 (2015), doi:10.1103/PhysRevE.91.022808

[16] Shiwakoti N, Sarvi M, Burd M: Using non-human biological entities to understand pedestrian crowd behaviour under emergency conditions. Safety Science vol. 66, pp. 1-8 (2014), doi:10.1016/j.ssci.2014.01.010

[17] Haghani M: Empirical methods in pedestrian, crowd and evacuation dynamics: Part I. Experimental methods and emerging topics. Safety Science vol. 129, pp. 104743-104743 (2020), doi:10.1016/j.ssci.2020.104743

[18] Muir HC, Bottomley DM, Marrison C: Effects of motivation and cabin configuration on emergency aircraft evacuation behavior and rates of egress. The International Journal of Aviation Psychology vol. 6(1), pp. 57-77 (1996), doi:10.1207/s15327108ijap0601_4

[19] Kretz T, Grünebohm A, Schreckenberg M: Experimental study of pedestrian flow through a bottleneck. Journal of Statistical Mechanics: Theory and Experiment vol. 2006(10), pp. 10014-10014 (2006), doi:10.1088/1742-5468/2006/10/P10014

[20] Daamen W, Hoogendoorn S: Emergency door capacity: influence of door width, population composition and stress level. Fire technology vol. 48(1), pp. 55-71 (2012), doi:10.1007/s10694-010-0202-9

[21] Chen JM, Lin P, Wu FY, Gao DL, Wang GY: Revisit the faster-is-slower effect for an exit at a corner. Journal of Statistical Mechanics: Theory and Experiment vol. 2018(2), pp. 023404-023404 (2018), doi:10.1088/1742-5468/aaa 8 f

[22] Shiwakoti N, Sarvi M: Enhancing the panic escape of crowd through architectural design. Transportation research part C: emerging technologies vol. 37, pp. 260-267 (2013), doi:10.1016/j.trc.2013.04.009 
[23] Lin P, Ma J, Liu T, Ran T, Si Y, Li T: An experimental study of the "faster-is-slower" effect using mice under panic. Physica A: Statistical Mechanics and its Applications vol. 452, pp. 157-166 (2016), doi:10.1016/j.physa.2016.02.017

[24] Shi X, Ye Z, Shiwakoti N, Tang D, Lin J: Examining effect of architectural adjustment on pedestrian crowd flow at bottleneck. Physica A: Statistical Mechanics and its Applications vol. 522, pp. 350-364 (2019), doi:10.1016/j.physa.2019.01.086

[25] Jianyu W, Jian M, Peng L, Juan C, Zhijian F, Tao L, et al.: Experimental study of architectural adjustments on pedestrian flow features at bottlenecks. Journal of Statistical Mechanics: Theory and Experiment vol. 2019(8), pp. 083402-083402 (2019), doi:10.1088/1742-5468/ab3190

[26] Sun L, Luo W, Yao L, Qiu S, Rong J: A comparative study of funnel shape bottlenecks in subway stations. Transportation Research Part A: Policy and Practice vol. 98, pp. 14-27 (2017), doi:10.1016/j.tra.2017.01.021

[27] Tavana H, Aghabayk K: Insights toward efficient angle design of pedestrian crowd egress point bottlenecks. Transportmetrica A: transport science vol. 15(2), pp. 1569-1586 (2019), doi:10.1080/23249935.2019.1619200

[28] Oh H, Park J: Main factor causing "faster-is-slower" phenomenon during evacuation: rodent experiment and simulation. Scientific reports vol. 7(1), pp. 1-14 (2017), doi:10.1038/s41598-017-14007-6

[29] Pan H, Zhang J, Song W: Experimental study of pedestrian flow mixed with wheelchair users through funnel-shaped bottlenecks. Journal of Statistical Mechanics: Theory and Experiment vol. 2020(3), pp. 033401-033401 (2020), doi:10.1088/1742-5468/ab6b1c

[30] Boyce K: Safe evacuation for all-Fact or Fantasy? Past experiences, current understanding and future challenges. Fire Safety Journal vol. 91, pp. 28-40 (2017), doi:10.1016/j.firesaf.2017.05.004

[31] Zhang T, Huang S-S, Zhang X-L, Lu S-X, Li C-H: Effect of exit location on flow of mice under emergency condition. Chinese Physics B vol. 28(1), pp. 010505010505 (2019), doi:10.1088/1674-1056/28/1/010505

[32] Wu F, Lin P, Gao D, Wang Z, Wang K, Ma J: An experimental study of exit position on escape efficiency using mice under competition. Journal of Statistical Mechanics: Theory and Experiment vol. 2019(1), pp. $013405-013405$ (2019), doi:10.1088/1742-5468/aaeee2

[33] Ezaki T, Yanagisawa D, Nishinari K: Pedestrian flow through multiple bottlenecks. Physical Review E vol. 86(2), pp. 026118-026118 (2012), doi:10.1103/PhysRevE.86.026118 
[34] Parisi DR, Patterson GA: Influence of bottleneck lengths and position on simulated pedestrian egress. Papers in Physics E vol. 9, pp. 090001-090001 (2017), doi:10.4279/PIP.090001 